\title{
EFEITO DA IDADE DA MATRIZ E DA TEMPERATURA DE ALOJAMENTO SOBRE A ABSORÇÃO DO SACO VITELINO E DESEMPENHO ZOOTÉCNICO DE FRANGOS DE CORTE
}

\author{
(Effect of breeder's age and temperature of first week of chicken in relation to retained yolk \\ sac and performance in broilers)
}

${ }^{1 *}$ Alexandre Teixeira Zocche, ${ }^{1}$ Cleverson de Souza, ${ }^{2}$ Clovis Eliseu Gewehr

\author{
${ }^{1}$ Correspondência: ${ }^{1}$ Pós-graduandos no Programa de Pós-graduação em Ciência Animal da Universidade do Estado de Santa Catarina \\ (UDESC) \\ ${ }^{2}$ Professor do Departamento de Produção Animal - UDESC/orientador \\ *zocche_alexandre_teixeira@elanco.com
}

RESUMO: A idade da matriz e a temperatura ambiental podem ser fatores determinantes na absorção do saco vitelino pelas aves e consequentemente no desempenho durante a criação. Com o objetivo de avaliar a absorção do saco vitelino nos sete primeiros dias de vida e o desempenho zootécnico de pintainhos oriundos de matrizes de idades diferentes alojados em diferentes temperaturas, um experimento foi conduzido no aviário do setor de Avicultura CAV/UDESC, durante 42 dias. Foram utilizados, 1.080 frangos Cobb machos de um dia de vida, originários de matrizes de 27, 37 e 60 semanas de idade, alojados em 30 boxes, submetidos a dois limites de temperatura, 30 a $32{ }^{\circ} \mathrm{C}$ e 24 a $26{ }^{\circ} \mathrm{C}$. As aves foram distribuídas em um delineamento inteiramente casualizado em arranjo fatorial $3 \times 2$ (idade da matriz $x$ temperatura), totalizando seis tratamentos, com cinco repetições de 36 aves/cada. Foram realizados quatro abates após a eclosão: antes do alojamento, 60, 108 e $156 \mathrm{~h}$, para colheita do peso de saco vitelino, fígado, proventrículo, bursa, baço e coração em quatro aves por repetição, sendo os valores expressos em \% em relação ao peso vivo. Aos 42 dias de idade duas aves de cada repetição foram sacrificadas para obtenção dos valores de peso relativo de órgãos, rendimento de carcaça e de cortes. Também foram avaliados semanalmente o consumo de ração, peso vivo e conversão alimentar. Os resultados foram submetidos à análise de variância e as médias avaliadas através do teste de Tukey (5\%). Matrizes mais velhas geram pintainhos mais pesados $(P<0,05)$ e influenciam $(P<0,05)$ as porcentagens dos órgãos na primeira semana, no entanto não afetam $(P<0,05)$ a absorção do saco vitelino. A temperatura de alojamento não influenciou as variáveis dos abates da primeira semana. Matrizes mais velhas geram frangos mais pesados $(P<0,05)$ aos 42 dias com maior, $(P<0,05)$, consumo de ração, porém, não influencia $(P>0,05)$ conversão alimentar, $\%$ de órgãos e rendimento de carcaça e de cortes. A temperatura de alojamento não influenciou $(P>0,05)$ o peso vivo, conversão alimentar, \% de órgãos $e$ rendimento de carcaça e de cortes das aves aos 42 dias, no entanto, aves criadas em temperatura abaixo do conforto térmico aumentam $(P<0,05)$ o consumo de ração na última semana de vida. Conclui-se que o desempenho de frangos de corte e os \% de órgãos são influenciados pela idade da matriz, enquanto que a temperatura de alojamento entre 24 a $26^{\circ} \mathrm{C}$ não afeta desempenho e peso relativo de órgãos. A idade de matriz e temperatura de 24 a $26{ }^{\circ} \mathrm{C}$ não influencia a absorção do saco vitelino.

Palavras-chave: Avicultura. Estresse térmico. Rendimento de carcaça 
ABSTRACT: The breeder's age and environment temperature can be an important factor to retained yolk for birds and consequently at performance during life of the chicken. The aim was evaluate the retained yolk in the first seven days of bird's life and zootechnical results of chickens from different breeder's age raising in different temperature. A trial was conducted in the experimental flock of Poultry department CAV/UDESC, during 42 days. There were used 1080 broilers Cobb males from three different breeder's age, been 360 chickens from each breeder's age staying birds in 30 boxes submitted two different temperature zone $30-32{ }^{\circ} \mathrm{C}$ and $24-26^{\circ} \mathrm{C}$. The bird were distributed in a randomized distribution at $3 \times 2$ factorial design (breeder's age $x$ temperature), totaling 06 treatment with 05 replicates of 36 birds/each. There were made four slaughter post hatch: before accommodation, 60 , 108 and 156 hours to collect and weight yolk, liver, pro ventricle, bursa, spleen and heart in four birds per replication, and the value expressed in percentage regardless live weight. 42 days old two birds was sacrificed per replication to obtain the organs weight, carcass yield, and cuts. Also there were evaluated weekly the feed intake, weight and feed conversion. The results were submitted the analyze of variance and average through test of Tukey (5\%). Older breeders produce heavy chicks $(P<0,05)$ and influence $(P<0,05)$ the percentage of organs at first week, however it does not affect $(P<0,05)$ the absorption of yolk. The temperature of brooders does not influence the variation of slaughter of first week. Older breeders produce broilers heaviest $(P<0,05)$ at 42 days with better $(P<0,05)$ feed intake, however it does not influence $(P<0,05)$ feed conversion, percentage of organs and carcass yield and cuts. The temperature of brooders does not influence $(P<0,05)$ the weight, feed conversion, percentage of organs and carcass yield at 42 days, however, birds raised in low temperature of comfort zone increase $(P<0,05)$ the feed intake at the last week of the chicken's life. Concludes that the performance of broilers and percentage of organs are influence by breeder's age while the temperature of brooders between $24-26 \mathrm{C}$ does not affect the performance and weight organs. The breeder's age and temperature of 24-26C does not influence at yolk.

Key Words: Carcass yield. Heat stress. Poultry 


\section{INTRODUÇÃO}

Nos últimos anos observou-se um aumento significativo da população mundial, sendo que a expectativa populacional até 2.050 é alcançar 9 bilhões de habitantes, consequentemente, esse aumento acarretara um maior demanda de alimentos, impondo às lideranças globais o desafio de aumentar a produção agrícola de maneira sustentável (FAO, 2009). A solução para atender à demanda atual de alimentos em quantidade e qualidade satisfatórios é desafiadora (BONAMIGO; BONAMIGO; MOLENTO, 2012). A produção, especialmente no setor de aves e suínos, tem seguido uma tendência semelhante aos países desenvolvidos, qual seja, a produção em larga escala. A expansão dessa tendência para todos os setores pecuários terá grande importância na redução da pobreza e aumento da segurança alimentar (FAO, 2009).

A produção avícola mundial é notoriamente ponto de referência em tecnologia e em estudos que visam aumentar a produção de proteína animal. Neste tocante, estudos relativos a fatores que influenciam o desempenho de frangos de corte devem ser realizados periodicamente, afim de sanar possíveis problemas na produção atrelado ao aumento dos índices produtivos da cadeia avícola. Dentre esses fatores, tem-se dois que influenciam efetivamente 0 desenvolvimento das aves, a temperatura de alojamento e a idade das progenitoras, ambos em conjunto ou não, são determinantes para a vida pré e pós eclosão das aves.

$A$ idade das matrizes influencia efetivamente 0 peso dos ovos (BURNHAM et al., 2001; ZAKARIA; MIYAKI; IMAI, 1983) Consequentemente afeta o peso dos pintainhos. Pintainhos mais pesados à eclosão, são mais resistentes e possivelmente serão frangos mais pesados ao final do período de criação. A temperatura de alojamento, por sua vez, influência nos parâmetros de desenvolvimento da ave pós eclosão, aves recém eclodidas quando expostas a temperaturas amenas reduzem 0 desenvolvimento, o que consequentemente se reflete em um pior desempenho (KHAN et al., 2011). Para a ave nas primeiras horas de vida, e durante 0 final da incubação, o fornecimento de nutrientes se dá pela absorção do saco vitelino, se logo após a eclosão algum fator prejudicar a absorção do saco vitelino, isso pode gerar deficiência nutricional e perda de produtividade por toda a vida da ave.

Como a absorção do saco vitelino se dá aproximadamente nos quatro primeiros dias de vida (CHAMBLEE et al., 1992) é imprescindível minimizar todos os possíveis riscos pelas quais está absorção possa ser prejudicada.

Desta forma, o presente estudo tem o objetivo de avaliar o efeito da idade da matriz e a temperatura de alojamento sobre a absorção do saco vitelino. As porcentagens de órgãos de pintainhos e o desempenho de frangos de corte também foram avaliadas.

\section{MATERIAL E MÉTODOS}

O projeto "Efeito da idade da matriz e da temperatura de alojamento sobre a absorção do saco vitelino e desempenho zootécnico de frangos de corte" foi submetido e avaliado pelo Comitê de Ética em Experimentação Animal (CETEA) sendo o protocolo de aprovação número 01.57.14.

O experimento foi realizado no Aviário Experimental do Laboratório de Patologia Aviária CAV/UDESC. O galpão possuía $16,7 \times 6,8 \mathrm{~m}$, sendo divido em 30 box de 2,0 $\mathrm{m}^{2}$ (unidades experimentais).

O galpão experimental possui sistema de pressão positiva, com cortinas amarelas duplas e piso de concreto. 
Cada parcela continha um comedouro infantil (utilizado até os sete dias), um comedouro tubular e dois bebedouros tipo nipple. O aquecimento na fase inicial foi realizado utilizando campânula elétrica, sendo uma em cada box. Cada parcela recebeu $5 \mathrm{~kg}$ de maravalha/ $\mathrm{m}^{2}$, totalizando $10 \mathrm{~kg}$ no total, por parcela, distribuídas uniformemente sobre o piso de concreto.

Foram utilizados 1080 pintos de corte, de um dia, machos, da linhagem COBB $\AA$, vacinados contra doença de Marek, Gumboro e Bronquite Infecciosa. O experimento constou de seis tratamentos correspondendo a dois intervalos de temperatura, de 30 a $32{ }^{\circ} \mathrm{C}$ (conforto térmico) e entre 24 a $26^{\circ} \mathrm{C}$ (estresse térmico por frio) e três idades de matrizes, 27, 37 e 60 semanas de idade.

Os pintos foram transportados do incubatório logo após o nascimento para o local do experimento, transcorrido um tempo de cerca de $4 \mathrm{~h}$ entre a eclosão e 0 alojamento.

As temperaturas na primeira semana de alojamento foram monitoradas a cada duas horas através de um termômetro digital a lazer modelo GM 300 da marca Benetech ${ }^{\circledR}$. As temperaturas de cada unidade experimental eram reguladas periodicamente através da altura das campânulas elétricas. As temperaturas médias diárias de cada tratamento estão demonstradas na Figura 1.

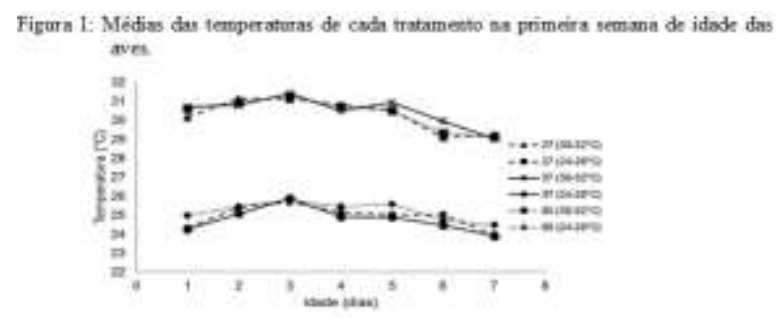

As rações experimentais, foram formuladas de acordo com as recomendações de Rostagno et al. (2011), para frangos de corte machos de desempenho médio, sendo apresentadas na Tabela 1.

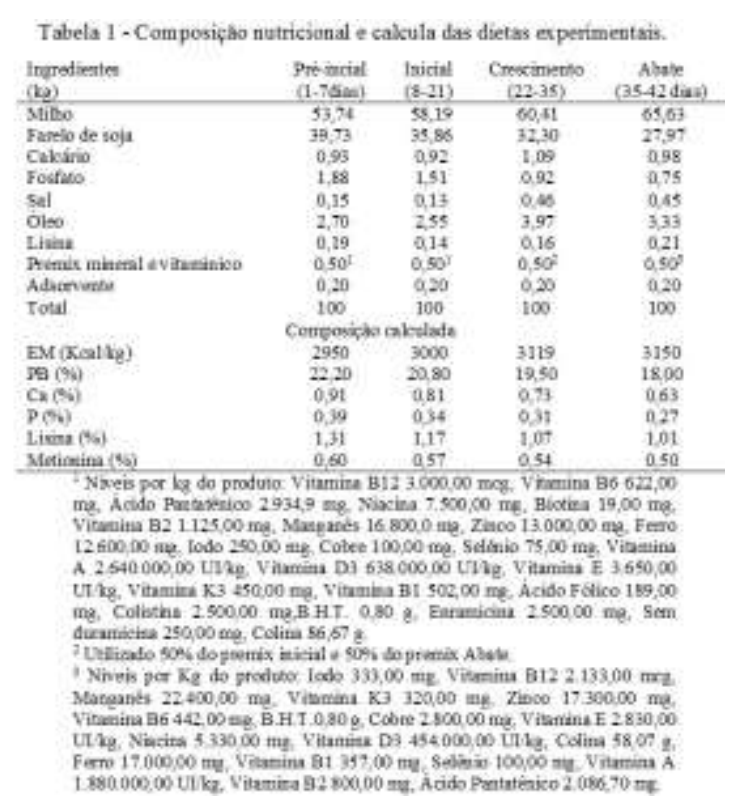

Antes do alojamento, as 60, 108, $156 \mathrm{~h}$ após a eclosão e aos 42 dias de vida, quatro aves por repetição, totalizando 20 aves por tratamento foram pesadas e sacrificadas para colheita dos dados de: peso do saco vitelino, fígado, proventrículo, bursa, baço e coração, e aos 42 dias foi avaliado o rendimento de carcaça e cortes. Durante o período experimental semanalmente foram coletados os dados de consumo de ração, peso vivo médio, ganho de peso médio e conversão alimentar.

O peso do saco vitelino e dos órgãos foi obtido através da remoção dos mesmos da cavidade visceral, com posterior pesagem em balança de precisão $(0,001 \mathrm{~g})$. Com o peso dos órgãos e da ave, foram calculados os índices de saco vitelino, fígado, proventrículo, bursa, baço e coração, dividindo-se o peso do órgão pelo peso vivo da ave, multiplicando por 100. O índice foi expresso em porcentagem.

O consumo semanal de ração de cada box foi obtido através do fornecimento prévio de uma quantidade de ração e ao final de cada semana, as sobras eram contabilizadas. O valor do consumo de cada box era dividido pelo número de aves do mesmo, obtendo-se consumo médio de ração por ave/dia. O consumo foi corrigido pela mortalidade. 
Peso vivo médio semanalmente as aves de cada box, eram pesadas coletivamente, e obtido o peso médio em quilogramas de cada ave.

Ganho de peso médio foi obtido através da relação entre o peso médio da parcela e a idade em dias, sendo expresso em g. Obtido semanalmente através da relação entre 0 consumo médio de ração $(\mathrm{kg})$ de cada box e 0 respectivo peso médio das aves $(\mathrm{kg})$.

Com 42 dias de idade, as aves foram submetidas a um jejum alimentar de $8 \mathrm{~h}$. Quatro aves por repetição foram separadas, levando em consideração o peso médio da parcela, para serem sacrificadas.

Após a colheita do peso vivo aves foram sacrificadas por deslocamento cervical seguido de sangria. Logo após realizouse a escaldagem em água a $60{ }^{\circ} \mathrm{C}$ e a depena por um minuto, aproximadamente. Procedeu-se a pendura, com a retirada da cloaca e evisceração.

A carcaça eviscerada, sem pés, pescoço e cabeça, foi pesada e posteriormente realizaram-se os cortes de peito e coxa/sobrecoxa, os quais foram pesados em balança de precisão $(0,001 \mathrm{~g})$. Obteve-se o rendimento de carcaça (\%) através da relação entre o peso da carcaça e o peso vivo da ave. Para o rendimento dos cortes considerou-se a relação entre o peso do mesmo e o peso vivo da ave.

As aves foram distribuídas em um delineamento inteiramente casualizado, em arranjo fatorial $2 \times 3$ (temperatura $x$ idade da matriz) com cinco repetições de 36 aves no dia do alojamento. Após os abates da primeira semana restaram 20 aves por unidade experimental para análise de desempenho aos 42 dias.

Os dados foram submetidos à análise estatística utilizando-se 0 programa estatístico, com nível de $5 \%$ de significância, para se descrever a influência da idade da matriz e a temperatura de alojamento. Os dados foram analisados por análise de variância levando em consideração a idade da matriz, a temperatura e a interação entre ambas. Quando significativo, foi desdobrado a interação. Quando não houve interação, as medias relacionadas a temperatura foram comparadas pelo teste $\mathrm{T}$ e as medias relacionadas a idade da matriz, comparadas pelo teste Tukey.

\section{RESULTADOS E DISCUSSÃO}

No primeiro abate (Tabela 2) as aves ainda não haviam sido submetidas ao tratamento com as temperaturas de alojamento, entretanto os dados referentes a esse tratamento indicam que as aves eram homogêneas ao início do experimento. Houve efeito $(P<0,001)$ da idade da matriz sobre o peso da ave e sobre $(P<0,05)$ a \% de coração. $O$ peso vivo das aves de matrizes com 60 semanas $(45,86 \mathrm{~g})$ foi maior $(\mathrm{P}<0,001)$ em relação as de 37 semanas $(43,22 \mathrm{~g})$ e 27 semanas $(36,81 \mathrm{~g})$. O mesmo ocorreu $(P<0,001)$ entre pintainhos de matrizes de 37 semanas e de 27 semanas, onde as mais velhas apresentaram proles mais pesadas.

No tocante a \% de coração, os pintainhos de matrizes de 60 semanas apresentam maiores $(P<0,05)$ valores em relação às matrizes mais jovens (27 semanas), no entanto não foi observada diferença $(P>0,05)$ entre matrizes de 60 e 37 semanas, e entre 37 e 27 semanas de idade.

Para os índices de saco vitelino, fígado, proventrículo, bursa, baço e coração não foram observados efeitos $(P>0,05)$ da idade da matriz.

Não foi observado interação $(P>0,05)$ entre temperatura de alojamento e a idade da matriz no primeiro abate (Tabela 2), isso se deve ao fato de que as aves ainda não haviam recebido o tratamento da temperatura de alojamento, já salientado anteriormente. Não foram observados efeitos $(P>0,05)$ da temperatura de alojamento sobre 0 
peso vivo e sobre os índices de saco vitelíno, fígado, proventrículo, bursa, baço e coração às $60 \mathrm{~h}$ de vida (Tabela 3).

Houve efeito $(P<0,001)$ da idade da matriz sobre o peso vivo, índice de fígado, bursa, baço e coração de frangos abatidos com $60 \mathrm{~h}$ de vida. Já para saco vitelíno e proventrículo não foram observados efeitos $(P>0,05)$ da idade da matriz.

Não houve interação $(P>0,05)$ entre os tratamentos às $60 \mathrm{~h}$ de vida.O peso vivo foi maior $(P<0,001)$ para frangos de matrizes de $60(65,07 \mathrm{~g})$ e $37(62,52 \mathrm{~g})$ semanas de idade em relação aos de matrizes de 27 semanas $(53,65 \mathrm{~g})$. Para índice de fígado, pintos de matrizes de 27 semanas tiveram maior valor $(4,59$ $\%)$ em relação as de matrizes de 37 semanas, $(4,28 \%)$, no entanto não diferiu $(P>0,05)$ de pintos de matrizes de 60 semanas $(4,48 \%)$, ocorrendo o mesmo com os de matrizes de 60 e 37 semanas.

Maior índice de bursa e baço foram observados $(P<0,05)$ em frangos de matrizes de 37 e 60 semanas em relação aos de 27 semanas. Os valores de bursa foram $0,18 \%$ e de baço 0,066 e $0,065 \%$ para os de 37 e 60 semanas respectivamente, enquanto que frangos de matrizes de 27 semanas tiveram \% de 0,13 para bursa e 0,046 para baço. No tocante a índice de coração, pintos de matrizes de 37 semanas apresentaram maiores $(P<0,05)$ valores $(0,96 \%)$ que os de matrizes de $60(0,87$ $\%)$ e $27(0,86 \%)$ semanas.

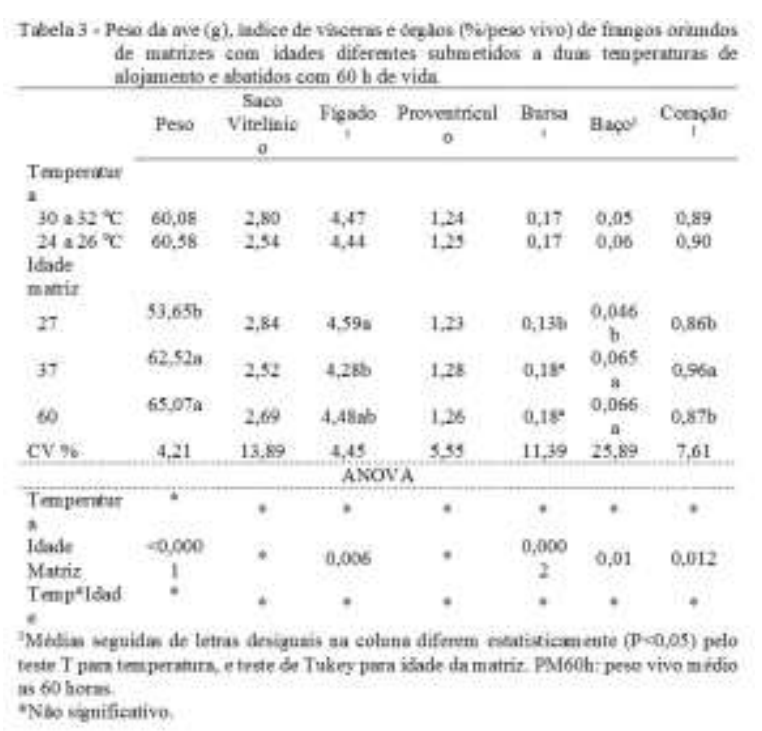

Não foram observados efeitos $(P>0,05)$ da temperatura de alojamento sobre o peso vivo e sobre os índices de saco vitelino, fígado, proventrículo, bursa, baço e coração às 108 horas de vida (Tabela 4).

Houve efeito $(\mathrm{P}<0,05)$ da idade da matriz sobre o peso vivo, índice de proventrículo e coração de frangos abatidos com $108 \mathrm{~h}$. Já para os índices de saco vitelino, fígado, bursa e baço não se observou efeitos $(P>0,05)$ da idade da matriz. Não houve interação $(P>0,05)$ entre os tratamentos.

$O$ peso vivo foi maior $(P<0,001)$ para frangos oriundos de matrizes com 60 e 37 semanas $(95,07$ e 91,02 g, respectivamente) em relação aos de matrizes de 27 semanas $(76,69 \mathrm{~g})$. Para o índice de proventículo, os pintainhos provenientes de matrizes com 27 semanas apresentam maior $(P<0,05)$ valor $(1,26 \%)$ em relação aos de aves com 60 semanas, $(1,19 \%)$, no entanto não diferiram aos de matrizes de 37 semanas $(1,22 \%)$. O mesmo ocorreu para os frangos de matrizes de 60 e 37 semanas, os quais não apresentaram diferenças $(P>0,05)$ entre si.

Maiores índices de coração foram observados $(P<0,05)$ em frangos de matrizes com 27 semanas (1,05\%) comparados a frangos de matrizes com 60 semanas $(0,96 \%)$, porém não diferiram $(P>0,05)$ de pintainhos 
oriundos de matrizes com 37 semanas $(1,01 \%)$. Para aves de matrizes com 37 e 60 semanas de idade não ocorreu efeito $(P>0,05)$ no índice de coração.

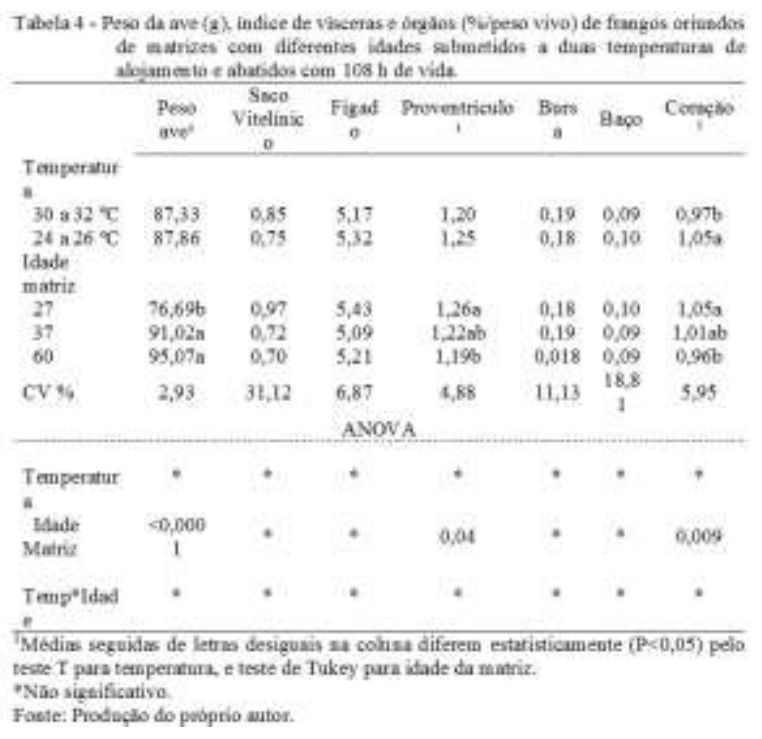

Não foram observados efeitos $(P>0,05)$ da temperatura de alojamento sobre o peso vivo e sobre índices de saco vitelínico, fígado, proventrículo, bursa, baço e coração às $156 \mathrm{~h}$ de vida (Tabela 5).

Houve efeito $(P<0,05)$ da idade da matriz sobre peso vivo, índice de proventrículo e baço de frangos abatidos com $156 \mathrm{~h}$ de vida. Já as porcentagens de saco vitelínico, fígado, bursa e coração não sofreram efeitos da idade da matriz (Tabela 5). Não houve interação $\quad(P>0,05)$ entre as temperaturas e idades da matriz.

$O$ peso vivo foi maior $(P<0,001)$ para frangos oriundos de matrizes com 60 $(127,28 \mathrm{~g})$ e $37(124,50 \mathrm{~g})$ semanas em relação as matrizes mais jovens, com 27 semanas, (107,84 g). Para o índice de proventículo, os pintainhos provenientes de matrizes com 27 semanas apresentam maior $(P>0,05)$ valor $(1,13 \%)$ em relação aos de aves com 37 semanas $(1,02 \%)$, no entanto não apresentaram diferença $(P>0,05)$ dos de matrizes com 60 semanas de idade (1,09\%), o mesmo ocorreu com matrizes de 60 e 37 semanas.
Maiores índices de baço foram observadas $(P<0,05)$ em frangos de matrizes com 27 semanas de idade $(0,12 \%)$ comparados a frangos de matrizes de 60 semanas, $(0,10 \%)$, porém não diferiram $(P>0,05)$ de pintainhos oriundos de matrizes com 37 semanas, $(0,11 \%)$.

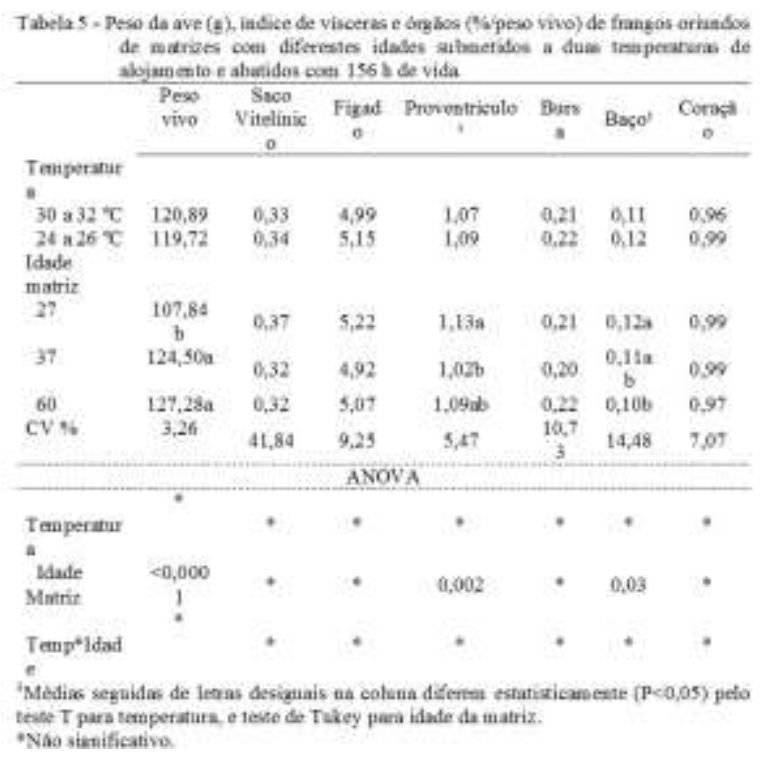

Muitos são os fatores que podem afetar o desenvolvimento e o desempenho de frangos de corte, dentre estes, os mais relevantes são a genética, nutrição, manejo, ambiência, peso do ovo e idade da matriz (DALANEZI et al., 2005; WILSON, 1991).

A medida que as matrizes envelhecem o período de ovulação das aves aumenta, acompanhado da redução na taxa de postura e aumento do peso do ovo. Esse aumento se deve ao fato de que a mesma quantidade de gema sintetizada via fígado é depositada em um número menor de folículos, elevando a proporção de gema no ovo (BURNHAM et al., 2001; ZAKARIA; MIYAKI; IMAI, 1983) e, consequentemente, o peso dos pintainhos. Evidenciou-se que cada grama de alteração no peso do ovo corresponde de 2 a $13 \mathrm{~g}$ de alteração no peso do frango de 6 a 8 semanas, sendo este efeito mais evidente em ovos de matrizes mais jovens comparadas com matrizes mais velhas 
(WILSON, 1991). No entanto, quando os ovos apresentam pesos semelhantes, não ocorre diferença entre o peso do pintainho em relação a idade da matriz (MCNAUGHTON et al., 1978).

Corroborando com os dados aqui demonstrados diversos autores (ALMEIDA et al., 2006; DALANEZI et al., 2005; FERNANDES et al., 2014; SINCLAIR; ROBINSON; HARDIN, 1990; ULMER-FRANCO; FASENKO; O'DEA CHRISTOPHER, 2010) relatam que matrizes mais velhas produzem ovos maiores, e consequentemente pintainhos maiores em relação a matrizes mais jovens.

Com relação ao saco vitelino, Vieira; Moran, (1998) relatam que não ocorreu diferença para essa variável entre matrizes com idade de 27 e 62 semanas de idade. No entanto, Fernandes et al., (2014) observaram efeito linear da idade da matriz sobre o índice de saco vitelino.

Ao eclodir um pintainho de $45 \mathrm{~g}$ tem aproximadamente $8 \mathrm{~g}$ de saco vitelino (NOY; SKLAN, 2001) que é composto por aproximadamente $47 \%$ de água, $42 \%$ de proteína, $52 \%$ de lipídios (MORAN; REINHART, 1980). Estes dados diferem aos obtidos neste experimento, pois o pintinho de maior peso, de 60 horas, resultou em 4,68 g. Provavelmente o tempo entre a eclosão de cerca de $4 \mathrm{~h}$ influenciou neste resultado.

Após a eclosão, a absorção do conteúdo da gema pode ser feita tanto pela membrana do saco vitelínico quanto pelo divertículo vitelínico, sendo digerido e absorvido pelo trato intestinal (ESTEBAN et al., 1991). Com base nos dados encontrados é possível afirmar que a idade da matriz e a temperatura de alojamento não influenciam a absorção do saco vitelino na primeira semana de vida dos frangos.

No abate de $60 \mathrm{~h}$ após a eclosão, a idade da matriz influenciou o índice de fígado, porém nos demais abates isso não foi observado. Como a gema tem aproximadamente $50 \%$ de lipídeos (NOY; UNI; SKLAN, 1996) e o metabolismo de lipídeos ocorre no fígado, isso pode ter influenciado 0 resultado. No entanto, após as $60 \mathrm{~h}$ de vida o índice de fígado se estabiliza, independentemente da idade.

O proventrículo juntamente com os outros órgãos do sistema digestório aumentam muito após a eclosão (UNI; FERKET, 2004). Este aumento não é acompanhado pelo peso da ave, visto que o índice de proventrículo é em ralação ao peso vivo da ave, aves mais pequenas apresentam um índice de proventrículo maior que pintainhos mais pesados, e isso está relacionado com a idade da matriz, como já citado anteriormente.

No tocante a índice de bursa e baço, os dados são contrários aos obtidos por El Sabry; Yalçin; Turgay-Izzetoğlu, (2013); Fernandes et al., (2014), os quais não observaram efeito da idade da matriz no peso dos órgãos em pintainhos. Como baço e a bursa são os principais órgãos linfoides do sistema imunitário, um índice mais elevado desses pode indicar uma maturação precoce dos mesmos (VAN DE VEN et al., 2013), sinalizando uma melhoria no sistema imune.

Com relação ao índice de coração, dados semelhantes foram observados por Barbosa et al., (2008); Luquetti et al. (2004), indicando aumento no peso absoluto do coração de pintos na eclosão a medida que aumenta a idade das matrizes. No entanto, neste trabalho foi observado que nos abates subsequentes, 60 e 108 h, o índice de coração oscilou conforme a idade da matriz. O que pode ter influenciado esse resultado é o peso do pintainho, pois o índice de coração é relacionado ao peso vivo, e como o coração não aumenta na mesma proporção do peso vivo, ocorre muita variação.

Não foram observados efeitos $(P>0,05)$ da temperatura de alojamento sobre 0 
peso médio aos sete (Pm7), quatorze (Pm14), vinte um (Pm21), vinte e oito (Pm28), trinta e cinco (Pm35) e quarenta e dois dias de idade (Pm42) dos frangos (Tabela 6). Em contra partida, a idade da matriz influenciou $(\mathrm{P}<0,001)$ o peso médio das aves durante todas as semanas de avaliação, onde pintainhos oriundos de matrizes com 37 e 60 semanas apresentaram pesos superiores aos pintainhos com de matrizes de 27 semanas (Tabela 6). Esse efeito se manteve desde os sete até os 42 dias de vida das aves.

Não foi observada interação $(P>0,05)$ entre a temperatura de alojamento e a idade da matriz para as variáveis analisadas, evidenciando que independentemente da idade da progenitora, a temperatura de alojamento não interfere no peso dos pintainhos.

Como relatado na literatura matrizes com idade intermediarias ou mais velhas produzem pintainhos mais pesados (Barbosa et al. 2008; El Sabry; Yalçin; Turgay-Izzetoğlu, 2013; Fernandes et al. 2014; Luquetti et al. 2004; Yalçin; Izzetoğlu; Aktaş, 2013). Como já salientado, a medida que as matrizes envelhecem o período de ovulação das aves aumenta, acompanhado da redução na taxa de postura e aumento do peso do ovo. Esse aumento se deve ao fato de que a mesma quantidade de gema sintetizada via fígado é depositada em um número menor de folículos, elevando a proporção de gema no ovo (BURNHAM et al., 2001; ZAKARIA; MIYAKI; IMAI, 1983) e consequentemente o peso dos pintainhos, sendo que esses frangos mais pesados à eclosão, mantiveram maior peso até os 42 dias de idade.

A temperatura de alojamento não influenciou o peso das aves, dados que descordam dos observados por Bruzual et al., (2000); Renwick; Washburn, (1982); Scott; Washburn, (1985), que encontraram redução do ganho de peso em aves que foram alojadas em temperaturas mais baixas. A redução na temperatura de alojamento pode afetar - comportamento dos pintainhos. Devido ao frio, as aves ficam agrupadas e imóveis, consequentemente ocorre uma redução na ingestão de água e alimento, podendo levar a desidratação (BRUZUAL et al., 2000). Entretanto, este fato não foi observado neste experimento.

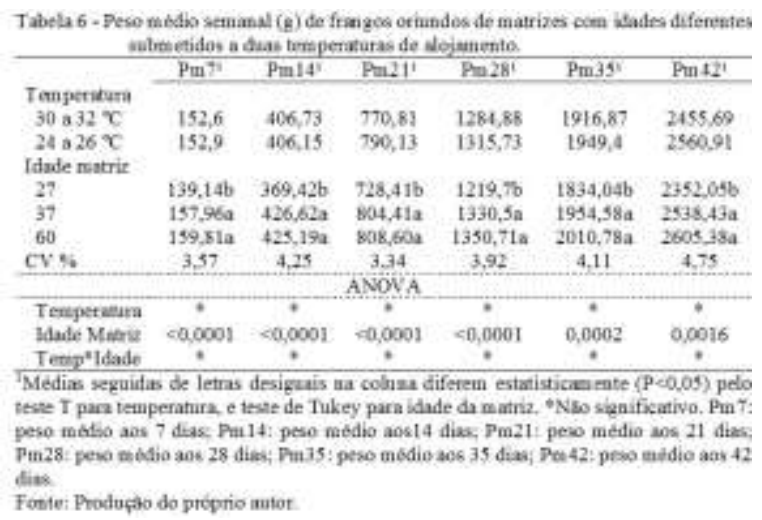

O consumo de ração semanal de frangos de corte submetidos a duas temperaturas de alojamento e provenientes de 3 idades de matriz estão na Tabela 7.

Não foram observados efeitos $(P>0,05)$ da temperatura de alojamento sobre 0 consumo médio do $1^{\circ}$ ao $7^{\circ}$ dia (Con7), do $8^{\circ}$ ao $14^{\circ}$ dia (Con14), do $22^{\circ}$ ao $28^{\circ}$ dia e do $29^{\circ}$ ao $35^{\circ}$ dia de idade dos frangos. No entanto, houve efeito $(\mathrm{P}<0,05)$ da temperatura de alojamento sobre o consumo durante o $15^{\circ}$ e $21^{\circ} \mathrm{e}$ durante o período compreendido entre $36^{\circ}$ e $42^{\circ}$ dia de idade das aves. Para as semanas em que houve efeito deste tratamento, as aves submetidas a uma menor temperatura de alojamento (estresse), consumiram maior $(P<0,05)$ quantidade de ração do que as criadas em conforto térmico.

A idade da matriz influenciou $(P<0,05) 0$ consumo semanal médio das aves até os 35 dias de vida. Na última semana (36 aos 42 dias) não se observou efeito $(P>0,05)$ da idade da matriz sobre 0 consumo. Até os 28 dias de idade, as 
aves provenientes de matrizes com idade de 27 semanas apresentaram menor $(P<0,05)$ consumo de ração do que filhos de matrizes com 37 e 60 semanas. No período que compreendeu dos 29 aos 35 dias, os frangos de matrizes com 27 e 37 consumiram menos $(P<0,05)$ ração que os oriundos de matrizes com 60 semanas. Não foi observada interação $(P>0,05)$ entre a temperatura de alojamento e a idade da matriz.

A ingestão de alimento afetada pela temperatura de alojamento corrobora com dados encontrados por Renwick; Washburn, (1982); Van der Pol et al., (2013) que observaram aumento $(\mathrm{P}<0,05)$ no consumo em aves que eram expostas a temperaturas mais amenas. Em temperatura mais amena, a ave ingere mais $(P>0,05)$ alimentos pois há um gasto maior de energia para manutenção da temperatura corporal.

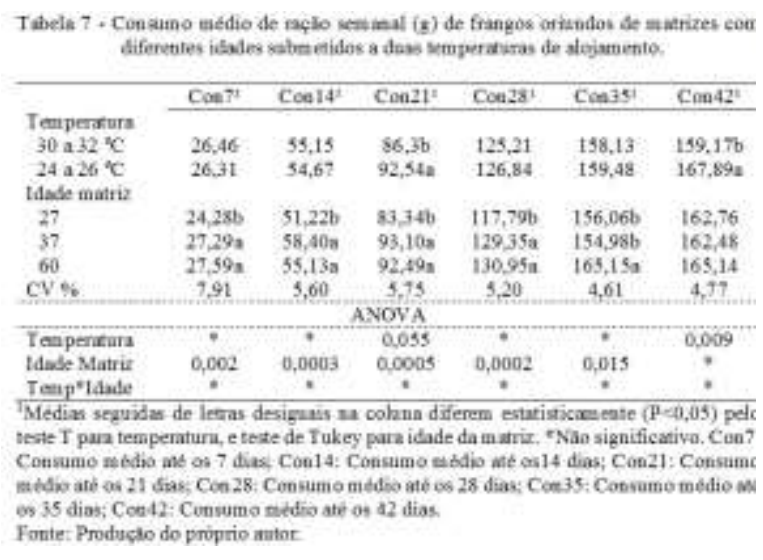

Não foram observados efeitos $(P>0,05)$ da temperatura de alojamento, idade da matriz e não ocorreu interação $(P>0,05)$ entre temperatura de alojamento e idade da matriz sobre a conversão alimentar em nenhuma semana durante 0 experimento (Tabela 8). Dados semelhantes foram relatados por May; Lott, (2000) que não observaram efeitos da temperatura sobre a conversão alimentar em frangos. No entanto Cerniglia; Hebert; Watts, (1983); Renwick; Washburn, (1982) observaram que aves criadas em temperaturas mais baixas tiveram uma maior conversão alimentar.

No que diz respeito a idade da matriz, os dados corroboram com os encontrados por Bruzual et al. (2000), que não observaram efeitos da idade da matriz sobre a conversão. Como a conversão alimentar é a relação entre consumo e peso das aves, filhos de matrizes mais velhas apresentam maior ganho de peso e consumo em relação aos pintainhos de matrizes jovens, no entanto a conversão alimentar mantevese inalterada.

Os dados de índices de fígado, baço, bursa, coração e rendimento de carcaça, peito e coxa-sobrecoxa, estão apresentados na Tabela 9.

A temperatura de alojamento e a idade da matriz não apresentou efeito $(P>0,05)$ sobre as variáveis aos 42 dias de idade. (DALANEZI et al., 2005). Também não foi encontrada interação entre os tratamentos para as variáveis em questão $(P>0,05)$. Dalanezi et al., (2005) observaram que filhos de matrizes jovens apresentam maior rendimento de peito que das aves velhas, no entanto não ocorre efeito sobre o rendimento de carcaça.

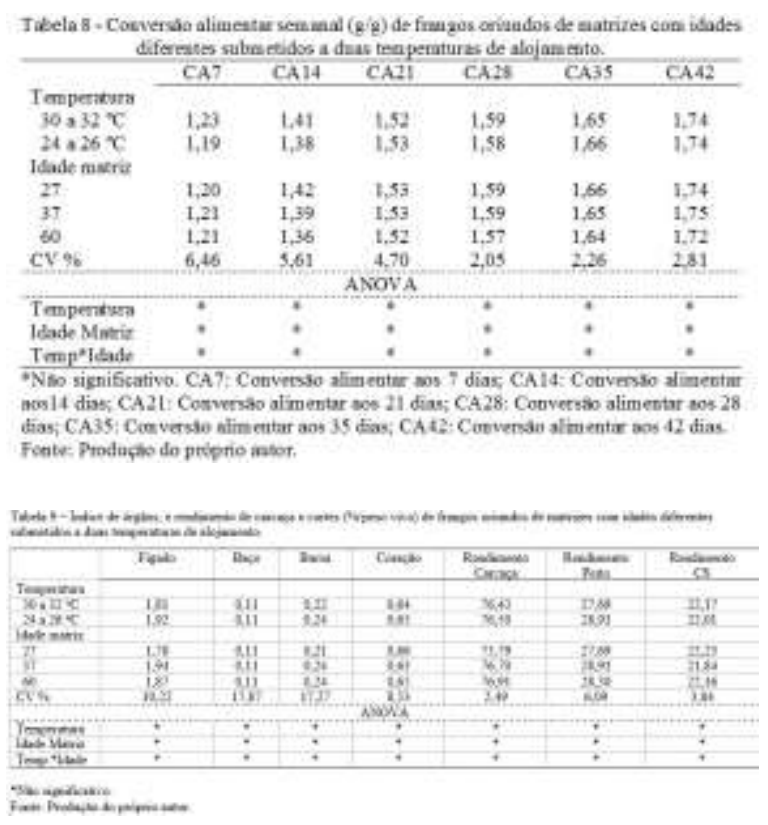




\section{CONCLUSÃO}

A temperatura de 24 a $26{ }^{\circ} \mathrm{C}$ não influencia os índices de órgãos na primeira semana, no entanto, altera o consumo de ração das aves durante a criação. A idade da matriz influencia os índices de órgãos de pintos na primeira semana e o desempenho de frangos de corte. Tanto as idades de matriz quanto as temperaturas testadas não influenciam a absorção do saco vitelino.

\section{NOTAS INFORMATIVAS}

O projeto "Efeito da idade da matriz e da temperatura de alojamento sobre a absorção do saco vitelino e desempenho zootécnico de frangos de corte" foi submetido e avaliado pelo Comitê de Ética em Experimentação Animal (CETEA) sendo o protocolo de aprovação número 01.57.14.

\section{REFERÊNCIAS}

ALMEIDA, J. G. et al.. Efeito da idade da matriz no tempo de eclosão, tempo de permanência do neonato no nascedouro e o peso do pintainho. Archives of Veterinary Science, v. 11, n. 1, p. 45-49, 2006.

BARBOSA, V. M. et al.. Efeitos da umidade relativa do ar na incubadora $e$ da idade da matriz leve sobre o rendimento da incubação. Arquivo Brasileiro de Medicina Veterinária e Zootecnia, v. 60, n. 3, p. 741-748, jun. 2008.

BONAMIGO, A.; BONAMIGO, C. B. DOS S. S.; MOLENTO, C. F. M. Atribuições da carne de frango relevantes ao consumidor: foco no bemestar animal. Revista Brasileira de Zootecnia, v. 41, n. 4, p. 1044-1050, abr. 2012.

BRUZUAL, J. J. et al.. Effects of relative humidity during the last five days of incubation and brooding temperature on performance of broiler chicks from young broiler breeders. Poultry science, v. 79 , n. 10, p. 1385-1391, 2000.

BURNHAM, M. R. et al.. Effects of incubator humidity and hen age on yolk composition in broiler hatching eggs from young breeders. Poultry science, v. 80, n. 10, p. 1444-1450, 2001.

CERNIGLIA, G. J.; HEBERT, J. A; WATTS, A B. The effect of constant ambient temperature and ration on the performance of sexed broilers. Poultry science, v. 62, n. 5, p. 746-754, 1983.

CHAMBLEE, T. N. et al.. Yolk sac absorption and initiation of growth in broilers. Poultry science, v. 71 , n. 11, p. 1811-1816, 1992.

DALANEZI, J. A. et al.. Efeito da idade da matriz sobre o desempenho e rendimento de carcaça de frangos de corte. Arquivo Brasileiro de Medicina Veterinária e Zootecnia, v. 57, n. 2, p. 250-260, abr. 2005.

EL SABRY, M. I.; YALÇIN, S.; TURGAY-IZZETOǦLU, G. Interaction between breeder age and hatching time affects intestine development and broiler performance. Livestock Science, $v$. 157, n. 2-3, p. 612-617, 2013.

ESTEBAN, S. et al.. A role played by the vitelline diverticulum in the yolk sac resorption in young post-hatched chickens. Journal of Comparative Physiology B, v. 160, n. 6, p. 645-648, 1991.

FAO, F. A. A. O.-. Livestock, food security and poverty reduction. In: The State of Food and Agriculture 2009: Livestock in the Balance. [s.l: s.n.]. p. 32-52.

FERNANDES, J. I. M. et al.. Influência da idade da matriz sobre a biometria de órgãos e a morfometria da mucosa do intestino delgado dos pintos à eclosão. Semina: Ciências Agrárias, v. 35, n. 2, p. 1083, 2014. 
LUQUETTI, B. et al.. Egg traits and physiological neonatal chick parameters from broiler breeder at different ages. Revista Brasileira de Ciência Avícola, v. 6 , n. 1, p. 13-17, mar. 2004.

MAY, J. D.; LOTT, B. D. The effect of environmental temperature on growth and feed conversion of broilers to 21 days of age. Poultry science, v. $79, \mathrm{n}$. 5, p. 669-671, 2000.

MCNAUGHTON, J. L. et al.. Effect of Age of Parents and Hatching Egg Weight on Broiler Chick Mortality. Poultry Science, v. 57, n. 1, p. 38-44, 1978.

MORAN, E. T.; REINHART, B. S. Poult yolk sac amount and composition upon placement: effect of breeder age, egg weight, sex, and subsequent change with feeding or fasting. Poultry science, v. 59, n. 7, p. 1521-1528, 1980.

NOY, Y.; SKLAN, D. Yolk and exogenous feed utilization in the posthatch chick. Poultry science, v. 80, n. 10, p. 1490-1495, 2001.

NOY, Y.; UNI, Z.; SKLAN, D. Routes of yolk utilisation in the newly-hatched chick. British poultry science, v. $37, \mathrm{n}$. 5, p. 987-995, 1996.

RENWICK, G. M.; WASHBURN, K. W. Adaptation of chickens to cool temperature Brooding. Poultry science, v. 61, n. 7, p. 1279-1289, 1982.

SCOTT, T. R.; WASHBURN, K. W. Evaluation of growth, hormonal, and hematological responses of neonatal chickens to reduced temperature brooding. Poultry science, v. 64, n. 5, p. 777-784, 1985.

SINCLAIR, R. W.; ROBINSON, F. E.; HARDIN, R. T. The Effects of Parent Age and Posthatch Treatment on Broiler Performance. Poultry Science, v. 69, n. 4, p. 526-534, 1990.

ULMER-FRANCO, A M.; FASENKO, G. M.; O'DEA CHRISTOPHER, E. E.
Hatching egg characteristics, chick quality, and broiler performance at 2 breeder flock ages and from 3 egg weights. Poultry science, v. 89 , n. 12 , p. 2735-2742, 2010.

UNI, Z.; FERKET, R. P. Methods for early nutrition and their potential. Worlds Poultry Science Journal, v. 60, n. 1, p. 101-111, 2004.

VAN DE VEN, L. J. F. et al.. Perinatal broiler physiology between hatching and chick collection in 2 hatching systems. Poultry science, v. 92, n. 4, p. 105061, 2013.

VAN DER POL, C. W. et al.. Effect of relative humidity during incubation at a set eggshell temperature and brooding temperature posthatch on embryonic mortality and chick quality. Poultry Science, v. 92, n. 8, p. 2145-2155, 1 ago. 2013.

VIEIRA, S. L.; MORAN, E. T. Eggs and chicks from broiler breeders of extremely different age. Journal of Applied Poultry Research, v. 7, n. 4, p. 372376, 1998.

WILSON, H. R. Reviews Interrelationships of egg size, chick size , posthatching growth and hatchability. World's Poultry Science Journal, v. 47, n. March, p. 5-20, 1991.

YALÇIN, S.; IZZETOĞLU, G. T.; AKTAŞ, A. Effects of breeder age and egg weight on morphological changes in the small intestine of chicks during the hatch window. British Poultry Science, v. 54, n. 6 , p. 810-817, 2013.

ZAKARIA, A H.; MIYAKI, T.; IMAI, K. The effect of aging on the ovarian follicular growth in laying hens. Poultry science, v. 62, n. 4, p. 670-674, 1983. 